\title{
Mechanisms and Clinical Implications of the Placebo Effect: Is There a Potential for the Elderly? A Mini-Review
}

\author{
Ulrike Bingel $^{\mathrm{a}}$ Luana Colloca $^{\mathrm{c}}$ Lene Vase $^{\mathrm{b}}$ \\ a Neurolmage Nord, Department of Neurology, University Medical Center Hamburg-Eppendorf,

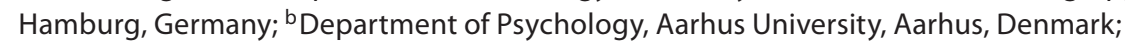 \\ 'National Center for Complementary and Alternative Medicine (NCCAM), Department of Bioethics, Clinical Center, \\ National Institutes of Health, Bethesda, Md., USA
}

\section{Key Words}

Placebo $\cdot$ Nocebo $\cdot$ Neurobiology $\cdot$ Placebo effect, clinical implications · Aging

\begin{abstract}
In recent years, the placebo effect has been a topic of considerable interest both in the scientific and the clinical community. In this time, the placebo effect has evolved from being considered a nuisance in clinical and pharmacological research to becoming a neurobiological phenomenon worthy of scientific investigation in its own right. Recent research shows that placebo effects are genuine psychobiological events attributable to the overall therapeutic context, and that these effects can be robust in both laboratory and clinical settings. These psychosocially induced biochemical changes in a patient's brain and body may in turn affect the course of a disease and the response to a therapy. Here we summarize and discuss the current insights into placebo mechanisms and discuss the potentially widespread implications for research and clinical practice. Even though a systematic knowledge of placebo effects across the lifespan is lacking, we aim at highlighting specific aspects related to the care of elderly patients and those suffering from neurodegenerative diseases.

Copyright $\odot 2010$ S. Karger AG, Basel
\end{abstract}

\section{KARGER}

Fax +41613061234

E-Mail karger@karger.ch

www.karger.com
(C) 2010 S. Karger AG, Basel

Accessible online at: www.karger.com/ger

\section{Introduction}

Placebo is derived from Latin and means 'I want to please' and originally placebo was conceptualized as a commonplace method or medicine prescribed in order to please the patient and not because of its efficiency $[1,2]$. Traditionally, placebo agents have been used as controls for active treatments in randomized clinical trials. Over the last decades, however, studies have shown that many of the patients who receive placebo treatments in randomized clinical trials experience symptom reduction $[3,4]$. Researchers have therefore become interested in investigating the context and disease-specific magnitude of placebo effects and their underlying mechanisms.

From the studies performed during the last decade, it has become evident that placebo effects represent complex neurobiological phenomena that are triggered by the psychosocial context of the patient and the therapy. It is important to point out that contextual and social stimuli may affect the patient's brain and body in many ways, and

All authors contributed equally to this work. Their names are listed in alphabetical order.

The opinions expressed by L.C. are those of the author and do not necessarily reflect the position or policy of the National Institutes of Health, the Public Health Service, or the Department of Health and Human Services.
PD Dr. Ulrike Bingel

NeuroImage Nord, Department of Neurology

University Medical Center Hamburg-Eppendorf

Martinistrasse 52, DE-20246 Hamburg (Germany)

Tel. +49 407410 53570, Fax +49 407410 59955, E-Mail bingel@ uke.uni-hamburg.de 


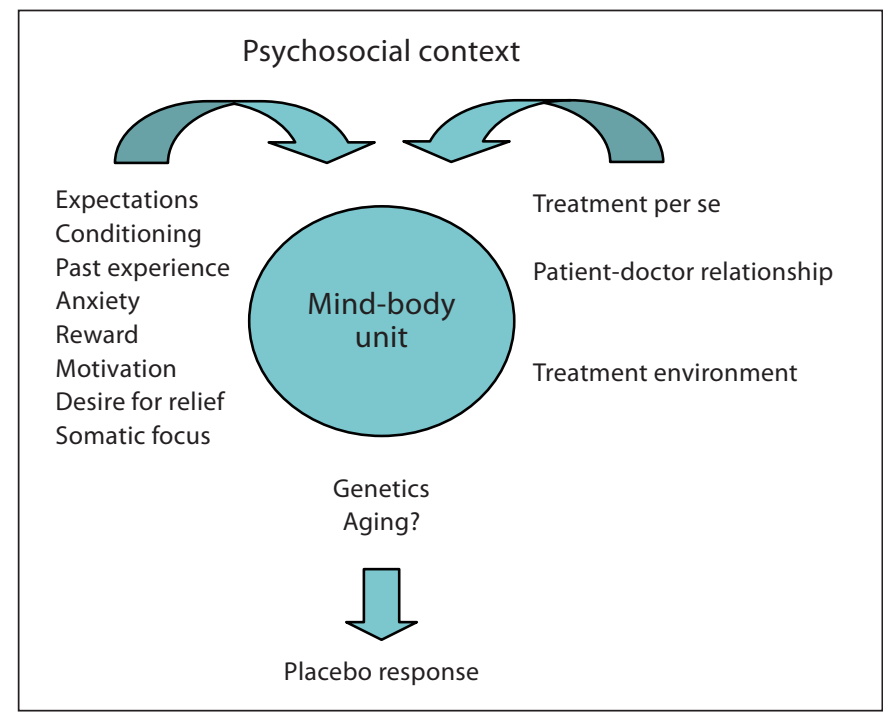

Fig. 1. Psychosocial context producing a placebo response. Left: Individual patient and clinician factors promoting the formation of a placebo response. Right: The treatment per se, the doctorpatient relationship and the treatment environment (e.g. therapeutic procedure, method of drug administration, technological devices) modulate the mind-body unit. Although speculative, genetic variability may influence the final outcome. Thus, individual, contextual and genetic factors interact with each other and determine the overall clinical placebo response.

hence it is not a matter of a single placebo effect. Rather, it seems that there are many, in part complementary, placebo effects that depend on the context, the affected system and the underlying disease. Although these studies have mostly been performed on younger age groups in the following, we will also present, speculatively at this point, the potential for clinical practice when providing care for older people. Moreover, we shall briefly discuss some examples of degenerative diseases and how these influence the formation of placebo responses.

\section{Contribution of Psychological Factors to Placebo Effects}

There are many factors that contribute to the generation of placebo effects (fig. 1). Environmental and psychosocial factors that have been described to influence placebo effects are verbal suggestion, expectation, meaning, conditioning, learning, reward, reduction of anxiety and behaviors and attitudes manifested by healthcare providers [5]. These factors must be assumed to vary greatly across clinical and research contexts, and consequently generate considerable variability in the placebo effect itself. Although there is a growing amount of research into these mechanisms, the two principal mechanisms that are best supported are expectancy and classical conditioning. For future research, it will be essential to explore if and how the mechanisms described below change during the lifespan, and how these different factors may be differentially affected/impaired in older patients or those suffering from degenerative diseases.

\section{Verbal Suggestion}

The verbal suggestions that the physician gives to the patients strongly influence the magnitude of placebo effects. Pollo et al. [6] investigated 38 thoractomized patients who were randomized to receive saline infusions along with three different types of suggestions. Patients in the first group received no information about the basal infusion and acted as a no-treatment condition. Patients in the second group were told that the infusion could be either a painkiller or a placebo, indicating a 50:50 chance for pain relief, whereas the third group of patients was told that the infusion was a powerful painkiller. Patients who were told that the agent was a powerful painkiller requested less medication than patients who were told that there was a 50:50 chance for pain relief, and the latter patients again requested less medication than the patients in the no-treatment condition. Hence, the stronger likelihood of pain relief, the greater the magnitude of the placebo analgesia effect was.

Similar findings are evident in the meta-analyses of placebo analgesia studies. When the placebo effect is investigated in clinical trials where patients have been told that they may receive either an active painkiller or an ineffective placebo agent, the magnitude of placebo analgesia effects is generally low (an effect size of $0.15-0.27$ Cohen's d) $[7,8]$. However, when the magnitude of placebo analgesia effects is investigated in studies of placebo mechanisms where patients are normally given strong verbal suggestions for pain relief, the magnitude of placebo analgesia effect is generally large (an effect size of 0.98-1.14, Cohen's d) $[8,9]$.

These findings have been further verified in two experimental studies of irritable bowel syndrome (IBS) patients who were exposed to rectal balloon distension and treated under no treatment, rectal placebo or rectal lidocaine conditions. The first study was conducted as a standard clinical trial where the patients were told that they 'may receive an active pain reducing medication or an 
inert placebo agent' [10]. This study found a significant pain-relieving effect of rectal lidocaine as compared to rectal placebo, and a significant pain-relieving effect of rectal placebo as compared to the natural history condition. The second study was conducted in a similar manner, the main difference being that in this study the patients were told 'the agent you have just been given is known to significantly reduce pain in some patients' [11]. A much larger placebo effect was found in the second study (an effect size of 2.0, Cohen's d), and the magnitude of the placebo analgesia effect was so high that there was no longer a significant difference between the magnitude of rectal lidocaine and rectal placebo. Hence, these two studies suggest that by adding an overt suggestion for pain relief it is possible to increase the magnitude of placebo analgesia to a level that matches that of an active agent.

\section{Expectation and Emotional Factors}

The verbal suggestions given for pain relief are likely to exert their influence through patients' expectations and emotional feelings. Expectancy is the experienced likelihood of an outcome or an expected effect, and within the context of pain studies expectancy can be measured by asking patients about the level of pain they expect to experience. Expectations are not influenced by verbal suggestions only but also by previous experiences via conditioning [12-14]. For example, different 'strengths' of treatment have been induced so that patients come to expect that treatment $\mathrm{A}$ is a strong painkiller and treatment $B$ is a weak painkiller, although both treatments are placebo treatments. By measuring the subjects' expectations immediately after the treatment has been given, it has become clear that the verbal suggestions given for pain relief influence patients' expectations of pain relief and that these expectations are closely related to the magnitude of the placebo effect [15].

Expectancies are likely modulated by emotion and vice versa. Accordingly, another psychological variable that has been suggested to contribute to placebo effects is the reduction of anxiety. This reduction seems to be especially true for placebo effects in the pain domain, where the pain-aggravating effect of anxiety is well characterized. This view is also supported by the neuroimaging data (see neurobiology paragraph) indicating reduced amygdala activity during the expectation of pain in the placebo condition. In the study of IBS patients mentioned above, patients' expectations for pain relief, their desire for pain relief and their anxiety levels were measured im- mediately after they had received either rectal placebo or rectal lidocaine treatment $[11,16]$. These variables including anxiety accounted for up to $80 \%$ of the variance in post-treatment pain ratings both in the placebo condition and in the active treatment condition. These findings indicate that expectations of pain relief and emotional factors such as reduction of anxiety are important placebo factors. In the clinical environment it is probably an interaction of both variables that determine the outcome of an individual placebo effect.

\section{Conditioning, Prior Experience}

Several studies have also investigated placebo effects by reinforcing expectations through a placebo manipulation in which the inert treatment is paired to lowered pain stimuli so that patients come to experience and expect pain relief $[12,17,18]$. This procedure typically evokes much stronger and more stable placebo analgesic effects compared to verbal suggestion alone and is therefore often used in experimental paradigms of placebo analgesia. Intriguingly, pharmacological conditioning has been shown to produce placebo responses that are largely independent of verbal suggestion or expectation, both in animals and humans.

Here, as a result of repeated associations between a neutral stimulus and an active drug (unconditioned stimulus), the neutral stimulus may become able to, by itself, elicit a response characteristic of the unconditioned stimulus. For instance, healthy volunteers who have repeatedly received an opioid infusion during an ischemic arm pain model will also develop analgesia when receiving a saline infusion that is explicitly given as a control condition [19]. Similar effects have also been observed in the motor, hormone and immune system [20]. Particularly fascinating are conditioned responses in the immune system, which seem not to be subject to voluntary control. Repeated associations between the immunosuppressive drug cyclosporin A (US, unconditioned stimulus), which inhibits both IL-2 and IFN- $\gamma$, and strawberry milk (CS, conditioned stimulus) induce conditioned responses in which the strawberry milk alone (without cyclosporin A) is capable of inhibiting both IL-2 and IFN- $\gamma$ [21].

It is worthy of note that conditioning can produce substantial placebo responses that will last several days. In fact, Colloca and Benedetti [18] observed that the exposure to effectiveness via conditioning elicited placebo analgesic responses that were present after a few minutes as well as after 4-7 days. In contrast, when the same condi- 
tioning procedure was repeated after a totally ineffective procedure, the placebo responses were remarkably reduced compared to the first group, pointing out that prior experience, both effective and ineffective, may have longlasting effects on the outcome of a subsequent treatment.

It is also interesting to evaluate the contribution of conditioning on nocebo modulation of pain. Nocebo hyperalgesia is a phenomenon that is the opposite of placebo analgesia. As for the placebo effect, the nocebo effect, or response, follows the administration of an inert substance (the nocebo, or negative placebo) along with the suggestion that the subject will get worse. In an experimental setting, Colloca et al. [17] found that negative conditioning and verbal suggestions induce equally significant nocebo hyperalgesic responses, whereas in the case of placebo analgesia, conditioning elicits larger reductions in pain than verbal suggestions alone. These findings suggest that nocebo responses may be elicited faster than placebo responses to protect the body from dangerous and negative outcomes.

\section{Role of Social Observational Learning}

Placebo analgesic effects might also occur without a history of actual first-hand experience because observation may convey information that is necessary to build up expectation of benefit. Colloca and Benedetti [22] found substantial placebo responses following observation of another subject undergoing a beneficial treatment. These responses were positively correlated with empathic concerns, suggesting that empathy may modulate observationally induced placebo analgesic responses. Interestingly, observation of a benefit in another person produced placebo responses that were similar in magnitude to those induced by directly experiencing the benefit through the conditioning procedure. Prosocial behaviors such as ability to share the other person's feelings, imitation and mimicry are critical in influencing psychophysical judgments of pain. These observations emphasize that the contextual cues and the whole atmosphere around the subject contribute to induce expectation of benefit and, thus, placebo responses.

\section{Is There a Placebo Personality?}

The responsiveness to placebos varies highly between individuals. It has long been known that there are placebo responders and non-responders. However, an unresolved

Mechanisms and Clinical Implications of the Placebo Effect issue is why some subjects respond to placebos while others do not. Also it is unclear how the individual placebo responsiveness varies for different biological systems and therapeutic contexts. Currently, there is a tremendous scientific and clinical interest in the identification of predictors of the individual placebo responding. Studies are being performed to characterize the role of psychological, physiological and genetic trait variables and their interaction with situational variables for placebo and nocebo phenomena.

\section{Gender}

To date, there is no conclusive evidence regarding the role of gender in relation to placebo responses. Gender effects in the placebo response were reported in an experimental setting with placebo analgesia during ischemic pain, in which males responded to the manipulation of expectancies through pain information while women did not [23]. However, an experimenter effect could not be ruled out in this study. Employing a motion-sickness paradigm, it has been observed that conditioning was more effective in women, while men exhibited a significantly greater reduction in rotation tolerance after verbal suggestions by responding more strongly to rotation and to suggestions than women [24].

\section{Personality}

For placebo analgesia a positive association with habitual optimism and state anxiety has been reported [25, 26]. An influence of suggestibility [15] has also been suggested. However, no conclusive data are available regarding the effect of personality traits on placebo responsiveness.

All in all, the probably interacting contribution of these various psychological factors across different physiological and pathological processes is far from being clear. Thus, to be able to make use of these mechanisms in clinical practice, it is crucial to further determine the relative contribution of each of them as possible sources of placebo and nocebo responses across different experimental and clinical conditions.

\section{Neurobiology of Placebo Effects}

Converging evidence from research since the 1970s substantiates that the placebo responses are not merely a psychological, but a complex psychoneurobiological phenomenon involving the activation of distinct brain areas as well as peripheral physiology including the release of endogenous substrates. 
Fig. 2. Impairment of placebo component in $\mathrm{AD}$ patients. Note the difference between normal subjects (on the left) and AD patients (on the right) as shown by electroencephalographic mutual information connectivity analysis, cognitive status assessed by means of frontal assessment battery (FAB), and pain reduction following the application of analgesic lidocaine according to the open-hidden paradigm. Note the difference between the connectivity peaks in a normal subject (top on the left) and an $\mathrm{AD}$ patient (top on the right). In $\mathrm{AD}$ patients, there was a reduction of brain connectivity, FAB scores, and psychosocial placebo component of the active medication. Modified from Colloca et al. [62]; data from Benedetti et al. [35].
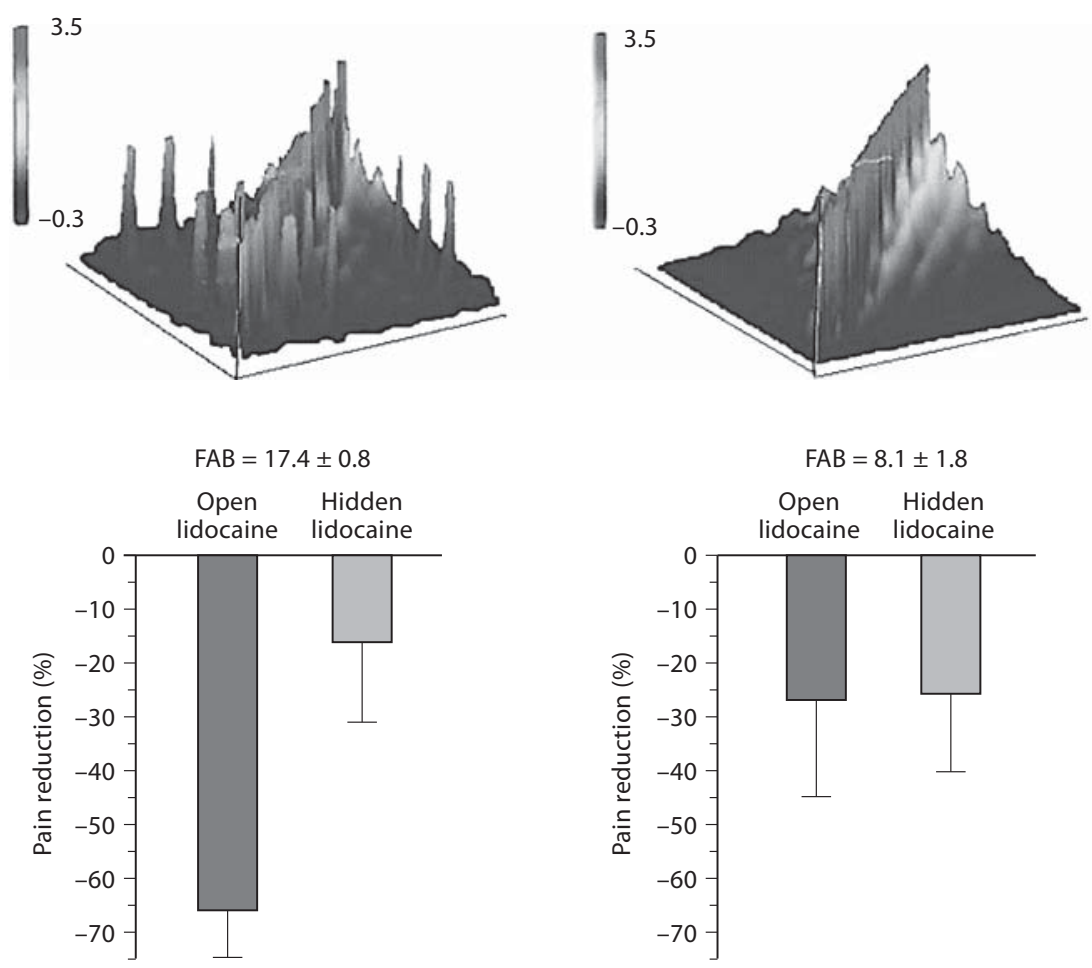

\section{Placebo Analgesia}

Placebo analgesia represents the best studied placebo response. Insights into its neurobiological mechanisms date back to the 1970s, when Levine et al. [27] discovered that placebo analgesia can be blocked by administering the opioid antagonist naloxone, which strongly suggested the involvement of endogenous opioid in its mechanism. In the past decade, functional brain imaging studies have contributed substantially to our knowledge of the brain mechanisms steering placebo phenomena.

\section{Placebo Analgesia Involves the Descending Pain Modulatory System}

The pioneering neuroimaging study further supported related mechanisms of opioid and placebo analgesia by revealing shared neural networks, including the rostral anterior cingulate cortex - the brainstem underlying both opioid- and placebo-related analgesia [28]. Since then, the relevance of this network has been substantiated by several studies using different procedures to induce placebo analgesia (including fake analgesic creams, sham acupuncture and others). All these studies demonstrate that placebo analgesia involves the activa- tion of cingulo-frontal brain regions together with subcortical structures such as the periaqueductal gray, hypothalamus and amygdala. Connectivity analyses further show that the behavioral placebo analgesic effect depends on an enhanced functional coupling of the frontal lobes and rostral anterior cingulate cortex with brainstem areas such as the periaqueductal gray [29-31]. These studies support the notion that placebo analgesia involves a top-down activation of endogenous analgesic activity via the descending modulatory system. The opioidergic nature of this pain-modulating system is supported by both pharmacological studies using the opioid antagonist naloxone and in vivo receptor-binding approaches [30, 32, 33]. The crucial relevance of the functional and structural integrity of cognitive-evaluative areas such as the prefrontal cortex with the downstream circuitry is emphasized by complementary experimental and clinical data: both the temporary functional lesion of the prefrontal cortex by repetitive transcranial magnetic stimulation [34] as well as the degeneration and disconnection of the frontal lobes in Alzheimer's disease (AD) [35] are associated with a reduction or complete loss of the placebo analgesic response 
(fig. 2). The clinical implications of these findings are discussed below. Overall, these findings strongly suggest that normal functioning of the prefrontal cortex is critical for triggering the descending pain control system and the placebo analgesic response.

\section{Placebo Analgesia and Activity in Sensory-Related}

Brain Areas

The majority of neuroimaging studies of placebo analgesia indicate that the reduced pain ratings during placebo analgesia are paralleled by decreased activity in the classical pain-processing areas including the thalamus, insula and somatosensory cortices $[29,31,36,37]$. This supports the notion that the altered pain experience during placebo analgesia results from active inhibition of nociceptive input and not simply from the reappraisal of otherwise unchanged input into the nociceptive brain or report bias. This, in turn, evokes the question of which level of the afferent nociceptive system this modulation occurs.

The latest advances in magnetic resonance physics and image analysis have made functional magnetic resonance imaging of the spinal cord technically feasible in both humans and animals. Recently, we used this technique to investigate the involvement of spinal cord processes in placebo analgesia. According to extensive work on descending pain modulatory mechanisms in animals, we hypothesized that placebo analgesia results in a reduction of nociceptive processing in the spinal cord [38]. Indeed, we found that pain-related activity in the ipsilateral dorsal horn, corresponding to painful stimulation, is strongly reduced under placebo. These results provided direct evidence for spinal inhibition as one mechanism of placebo analgesia and highlighted the fact that psychological factors can act on the earliest stages of pain processing in the central nervous system.

All these studies support the notion that placebo analgesia involves a top-down activation of endogenous analgesic activity via the descending modulatory system. The very same system targeted by exogenous opioid administration [39]. The additional contribution of emotional regulation processes and areas such as the limbic system is a fundamental step that needs to be further addressed. Most likely it is a combination of both these effects that contributes to the placebo-induced reduction in pain perception.

\section{Placebo in Anxiety and Depression}

The above-mentioned neural circuitry underlying placebo analgesia does not seem to be specific for the generation of placebo responses in the pain domain. Similar patterns of brain activations have also been observed in brain-imaging studies of emotional placebo using placebo anxiolytics or placebo antidepressants. Petrovic et al. [40] found a shared modulatory network involving the rostral anterior cingulate cortex and the lateral orbitofrontal cortex during both emotional placebo and placebo analgesia. These effects were correlated with the reported placebo effect and were predicted by the amount of treatment expectation. Similarly, therapy with placebo or the SSRI fluoxetine in patients with major depression had similar effects on brain metabolic changes in the anterior and posterior cingulate cortex and prefrontal cortex after 6 weeks of treatment [41]. Depressed patients who undergo a placebo treatment also showed distinct electroencephalographic patterns in the prefrontal region; these patterns were found by means of an off-line elaboration of EEG recordings (cordance method), a method developed in their laboratory [42]. In addition, serotonin-related gene polymorphisms have been found to be influence the individual placebo response in social anxiety, both at the behavioral and neural level (amygdala activity during a stressful public speaking task) [43] and genetic polymorphisms modulating monoaminergic tone (catabolic enzymes catechol-O-methyltransferase and monoamine oxidase A) have been related to degree of placebo responsiveness in major depressive disorder [44].

\section{Parkinson's Disease}

Analogously, studies investigating placebo responses in Parkinson's disease demonstrate that the expectationinduced improvement of motor functions following a sham treatment is associated with the release of endogenous dopamine in the basal ganglia. de la FuenteFernandez et al. [45] used raclopride-PET to study involvement of the endogenous dopamine system for placebo responses in Parkinson patients. Following the administration of a placebo that the patients believed to be apomorphine (a powerful anti-parkinsonian treatment), they observed increasing dopaminergic neurotransmission in the striatum, which corresponded with clinical improvement. Similarly, Benedetti et al. [46, 47] recorded changes in the firing patterns and bursting activity of neurons in the subthalamic nucleus and associated motor circuitry during the placebo response in Parkinson patients undergoing the implantation of deep brain stimulation.

However, the dopaminergic system is a complex system involved in a variety of brain functions, including 
Table 1. Mechanisms of placebo effects in medical conditions and physiological systems (adapted from Finniss et al. [2])

\section{Mechanisms}

\begin{tabular}{ll}
\hline Pain & $\begin{array}{l}\text { Activation of endogenous opioids and dopamine } \\
\text { (placebo); activation of cholecystokinin and } \\
\text { deactivation of dopamine (nocebo) }\end{array}$ \\
\hline
\end{tabular}

\begin{tabular}{ll}
\hline $\begin{array}{l}\text { Parkinson's } \\
\text { disease }\end{array}$ & $\begin{array}{l}\text { Activation of dopamine in the striatum and } \\
\text { changes in activity of neurons in basal ganglia, } \\
\text { and thalamus }\end{array}$
\end{tabular}

Depression Changes of electrical and metabolic activity in
different brain regions (e.g. ventral striatum)

\begin{tabular}{ll}
\hline Anxiety & $\begin{array}{l}\text { Changes in activity of the anterior cingulate and } \\
\text { orbitofrontal cortices; genetic variants of sero- } \\
\text { tonin transporter and tryptophan hydroxylase-2 }\end{array}$ \\
\hline Addiction & $\begin{array}{l}\text { Changes of metabolic activity in different brain } \\
\text { regions }\end{array}$ \\
\hline
\end{tabular}

Cardiovas- $\quad$ Reduction of $\beta$-adrenergic activity of heart

cular system

\begin{tabular}{ll}
\hline $\begin{array}{l}\text { Respiratory } \\
\text { system }\end{array}$ & $\begin{array}{l}\text { Conditioning of opioid receptors in the } \\
\text { respiratory centers }\end{array}$ \\
\hline $\begin{array}{l}\text { Immune } \\
\text { system }\end{array}$ & $\begin{array}{l}\text { Conditioning of immune mediators } \\
\text { (e.g. interleukin-2, interferon- } \gamma \text {, lymphocytes) }\end{array}$ \\
$\begin{array}{l}\text { Conditioning of antihistamine effects in allergic } \\
\text { rhinitis }\end{array}$
\end{tabular}

\begin{tabular}{ll}
\hline $\begin{array}{l}\text { Endocrine } \\
\text { system }\end{array}$ & $\begin{array}{l}\text { Conditioning of some hormones } \\
\text { (e.g. growth hormone, cortisol) }\end{array}$
\end{tabular}

reward mechanisms. This might be the reason why dopamine release during a placebo response is not specific for Parkinson's disease. Rather, dopaminergic mechanisms have been associated with placebo responses or placebolike phenomena in different systems, including the pain system [48]. The detailed context and condition-specific role of dopamine for placebo responses needs to be identified in future studies.

In summary, these studies, exemplarily reported for three different systems, strengthen the notion that placebo-induced clinical benefits involve system-specific neurobiological responses which are the very same as those targeted by specific pharmacological treatments. For an overview of placebo/nocebo effects in different physiological systems, see table 1 . However, the specificity of the involved circuitry and neurotransmitter systems is still unclear. Common and system-specific pathways steering placebo responses across different diseases need to be addressed in future research.

\section{Placebo Components in Active Pharmacological Treatment}

The recent scientific interest in placebo and nocebo phenomena has increased the awareness for the fact that also active pharmacological treatments, inevitably, comprise interacting physiological and psychosocial components. The crucial role of expectation in the therapeutic outcome is best illustrated in the so-called open/hidden drug paradigms. In these paradigms, identical concentrations of drugs are administered in two different conditions: an open condition, where the patient is aware of the medication being given by a healthcare provider who is also announcing the intended treatment outcome (e.g. analgesia), and a hidden condition, where the patient is unaware of the medication being administered by a computer-controlled infusion. This allows for dissociating the pharmacodynamic effect of the treatment (hidden treatment) and the additional benefit of the psychosocial context in which the treatment is given. The difference between the outcomes following the administration of the expected and unexpected therapy can be seen as the 'placebo' (psychological) component, even though no placebo has been given. These studies reveal that psychosocial factors such as awareness of a drug being given can considerably enhance the analgesic effect of a drug [49]. This phenomenon is not restricted to analgesics, as similar effects have also been reported for treatments in other domains, such as motor function in Parkinson's disease and anxiety-related disorders [50,51].

These behavioral observations highlight the crucial relevance of psychosocial effects to drug efficacy, yet we have little information about the neurobiological mechanisms by which psychological treatment effects combine with those of biologically active drugs. Given that psychological treatment effects (as the expectancy of treatment outcome) are shown to be mediated by the very same biological systems through which drugs exert their treatment effects, intricate interactions of pharmacological and psychosocial factors must be assumed to converge at the neurobiological level. Future experimental and clinical studies will need to explore these interactions in greater detail.

\section{Clinical Implications}

The improved understanding of the neuropsychology and neurobiology underlying placebo and nocebo effects holds potentially far-reaching implications for future re- 
search and clinical practice. Evidence from clinical studies emphasizes the crucial relevance of contextual variables, including physician-patient interactions, for treatment outcome [52-54]. Complementary experimental research further reveals that the psychosocial context induces biochemical changes in the patient's brain and body that in turn may affect the natural history of a disease and the response to a treatment. Along these lines, negative reactions to drugs have been shown, in most cases, much better predicted by the patient's individual beliefs and negative expectations regarding a drug's effect (nocebo effects) than by the specific pharmacological properties of the drug itself [55-57].

These findings should remind healthcare practitioners of the relevance of psycho-physiological interactions for medical practice and underpin the principal importance of patient-physician interactions, the key to influence the patients' concepts of disease, treatment expectancies and their ability to cope with therapeutic side effects. However, this knowledge is at odds with the observation that in the United States, $50 \%$ of all patients leave after an office visit without an adequate understanding of what the physician has told them [58].

A recent study aimed at identifying variables that predict the response to an experimentally applied supportive patient-practitioner relationship in IBS patients. Intriguingly, out of 452 baseline variables, only two factors increased sensitivity to the supportive patient-practitioner relationship, one of them being reclusiveness [59]. This suggests that elderly patients, who often live alone, would especially benefit from increased and intensified care by healthcare practitioners.

Even though aging involves substantial changes in many systems known to be involved in placebo responses, age-related changes in placebo responding have not, to the best of our knowledge, been addressed systematically. However, both clinical and experimental data support the notion that elderly patient groups are generally able to produce placebo effects. For example, clinical trials of antidepressants for late life depression reveal response rates in the placebo arm between 40 and $60 \%$. These cannot be entirely be related to the natural time course of the disease (e.g. remission of depression), as the placebo response rates depended on the trial designs thus on contextual variables [60]. Although the boundary between middle age and old age cannot be defined exactly, clinical and experimental research shows that elderly patients can also manifest placebo effects detectable at the neurobiological level. de la Fuente-Fernandez et al. [45] and Benedetti et al. [46] reported a placebo-induced dopamine release in parkinsonian patients with an age range of 45-74 years (average 58) and 50-75 years (average 62).

However, it is important to note that specific age-associated diseases can substantially impair the ability to produce placebo responses. The impairment of cognitive functions has been found to disrupt the psychosocial component of a treatment. Benedetti et al. [35] applied a local anesthetic, either overtly or covertly, to the skin of patients with $\mathrm{AD}$ to reduce burning pain after venipuncture [35]. They found that AD patients (mean age $73.5 \pm$ 6.8 years) with reduced prefrontal executive control and decreased EEG connectivity of the prefrontal lobes with the rest of the brain, showed a reduced placebo component of the analgesic treatment. Remarkably, the loss of these placebo-related mechanisms reduced treatment efficacy, so that a dose increase was necessary to produce adequate analgesia. This represents the first study of loss of expectation of benefit and impairment of placebo mechanisms in a clinical condition (fig. 2). Interestingly, the study included a control group with old healthy volunteers (mean age $70 \pm 6.1$ years) showing robust placebo analgesic responses, suggesting that psychobiological mechanisms modulate the effectiveness of active treatments in older people.

However, it does not need a neurobiological foundation as in $\mathrm{AD}$ to disturb the balance of pharmacological and psychosocial treatment components: expectation and prior experience are key psychological mechanisms of placebo and nocebo responses. Treatment expectations and experiences with physicians and treatments are ubiquitous in patients, particularly in those with chronic conditions. Negative and frustrating treatment experiences multiply throughout the lifespan and the course of diseases and shape the expectancy for future treatments. Furthermore, the accompanying negative mood states, especially in chronic patients [61], lend themselves to generating negative treatment expectations. In these situations, drugs with biologically plausible intrinsic actions compete with the negative treatment expectancies of the patient, expectations that can modulate or, in the worst case, completely abolish their effects.

We propose that it is essential to harness psychosocial and contextual mechanisms alongside traditional treatment considerations to optimize pharmacotherapy. This might be especially beneficial for elderly patients and for those who are chronically ill and hence characterized by a constellation of polypharmacotherapy and increased risk of adverse events due to reduced drug tolerance. It will be necessary to consider the contribution of negative 
experience to drug efficacy, especially in elderly patients whose experience with treatments and physicians has naturally piled up along the lifespan. Also the high prevalence of comorbidity with diseases like depression, anxiety or dementia that potentially impair drug efficacy by an impairment of placebo effects or, what is worse, the adding of nocebo effects warrants special attention and care in old patients.

Our current understanding of the role of psychosocial components in relation to drug efficacy has implications for clinical trial designs as well. We propose that influencing beliefs about outcome by careful use of language (or other cognitive-behavioral approaches) should be considered a new frontier of pharmacological management. Rather than seeking to control away psychological treatment components, new trial designs could be developed that aim to maximize the pharmacological effects and to minimize side effects of therapeutic agents by un- derstanding the role of the context in which a drug is given. A new appreciation of the role of individual differences (genetically, psychologically, and neurologically) that includes an investigation of the patient's age and treatment history represents to us an exciting possibility for personalized medicine that promises to optimize treatment outcome.

\section{Acknowledgements}

This work was supported by the BMBF (U.B.), Regione Piemonte and International Association for Study of Pain (L.C.) and ViFAB (L.V.).

\section{Disclosure Statement}

The authors declare no conflicts of interest.

\section{References}

1 Shapiro AK, Shapiro E: The Powerful Placebo: From Ancient Priest to Modern Physician. Baltimore, Johns Hopkins University Press, 1997.

-2 Finniss DG, Kaptchuk TJ, Miller F, Benedetti F: Biological, clinical, and ethical advances of placebo effects. Lancet 2010;375:686-695

3 Beecher HK: The powerful placebo. JAMA 1955;159:1602-1606.

4 Walsh BT, Seidman SN, Sysko R, Gould M: Placebo response in studies of major depression: variable, substantial, and growing. JAMA 2002;287:1840-1847.

5 Price DD, Finniss DG, Benedetti F: A comprehensive review of the placebo effect: recent advances and current thought. Annu Rev Psychol 2008;59:565-590.

-6 Pollo A, Amanzio M, Arslanian A, Casadio C, Maggi G, Benedetti F: Response expectancies in placebo analgesia and their clinical relevance. Pain 2001;93:77-84.

7 Hrobjartsson A, Gotzsche PC: Is the placebo powerless? An analysis of clinical trials comparing placebo with no treatment. N Engl J Med 2001;344:1594-1602.

8 Vase L, Riley JL, Price DD: A comparison of placebo effects in clinical analgesic trials versus studies of placebo analgesia. Pain 2002;99:443-452.

-9 Vase L, Petersen GL, Riley JL 3rd, Price DD: Factors contributing to large analgesic effects in placebo mechanism studies conducted between 2002 and 2007. Pain 2009; 145: $36-44$.
10 Verne GN, Robinson ME, Vase L, Price DD: Reversal of visceral and cutaneous hyperalgesia by local rectal anesthesia in irritable bowel syndrome patients. Pain 2003;105: 223-230.

11 Vase L, Robinson ME, Verne GN, Price DD: Increased placebo analgesia over time in irritable bowel syndrome patients is associated with desire and expectation but not endogenous opioid mechanisms. Pain 2005;115: 338-347.

12 Voudouris NJ, Peck CL, Coleman G: Conditioned response models of placebo phenomena: further support. Pain 1989;38:109-116.

13 Montgomery GH, Kirsch I: Classical conditioning and the placebo effect. Pain 1997;72: 107-113.

14 Klinger R, Soost S, Flor H, Worm M: Classical conditioning and expectancy in placebo hypoalgesia: a randomized controlled study in patients with atopic dermatitis and persons with healthy skin. Pain 2007;128:31-39.

15 De Pascalis V, Chiaradia C, Carotenuto E: The contribution of suggestibility and expectation to placebo analgesia phenomenon in an experimental setting. Pain 2002;96: 393-402.

16 Vase L, Robinson ME, Verne GN, Price DD: The contributions of suggestion, desire, and expectation to placebo effects in irritable bowel syndrome patients. An empirical investigation. Pain 2003;105:17-25.

17 Colloca L, Sigaudo M, Benedetti F: The role of learning in nocebo and placebo effects. Pain 2008;136:211-218.
18 Colloca L, Benedetti F: How prior experience shapes placebo analgesia. Pain 2006; 124 : 126-133.

19 Amanzio M, Benedetti F: Neuropharmacological dissection of placebo analgesia: expectation-activated opioid systems versus conditioning-activated specific subsystems. J Neurosci 1999;19:484-494.

20 Benedetti F, Pollo A, Lopiano L, Lanotte M, Vighetti S, Rainero I: Conscious expectation and unconscious conditioning in analgesic, motor, and hormonal placebo/nocebo responses. J Neurosci 2003;23:4315-4323.

21 Goebel MU, Trebst AE, Steiner J, Xie YF, Exton MS, Frede S, Canbay AE, Michel MC, Heemann U, Schedlowski M: Behavioral conditioning of immunosuppression is possible in humans. FASEB J 2002;16:18691873.

22 Colloca L, Benedetti F: Placebo analgesia induced by social observational learning. Pain 2009; 144:28-34.

23 Flaten MA, Aslaksen PM, Finset A, Simonsen $\mathrm{T}$, Johansen O: Cognitive and emotional factors in placebo analgesia. J Psychosom Res 2006;61:81-89.

24 Klosterhalfen S, Kellermann S, Braun S, Kowalski A, Schrauth M, Zipfel S, Enck P: Gender and the nocebo response following conditioning and expectancy. J Psychosom Res 2009;66:323-328.

25 Geers AL, Kosbab K, Helfer SG, Weiland PE, Wellman JA: Further evidence for individual differences in placebo responding: an interactionist perspective. J Psychosom Res 2007; 62:563-570. 
-26 Morton DL, Watson A, El-Deredy W, Jones AK: Reproducibility of placebo analgesia: effect of dispositional optimism. Pain 2009; 146:194-198.

-27 Levine JD, Gordon NC, Jones RT, Fields HL: The narcotic antagonist naloxone enhances clinical pain. Nature 1978;272:826-827.

-28 Petrovic P, Kalso E, Petersson KM, Ingvar M: Placebo and opioid analgesia - imaging a shared neuronal network. Science 2002;295: 1737-1740.

29 Wager TD, Rilling JK, Smith EE, Sokolik A, Casey KL, Davidson RJ, Kosslyn SM, Rose RM, Cohen JD: Placebo-induced changes in fMRI in the anticipation and experience of pain. Science 2004;303:1162-1167.

-30 Eippert F, Bingel U, Schoell ED, Yacubian J, Klinger R, Lorenz J, Buchel C: Activation of the opioidergic descending pain control system underlies placebo analgesia. Neuron 2009;63:533-543.

- 31 Bingel U, Lorenz J, Schoell E, Weiller C, Buchel C: Mechanisms of placebo analgesia: rACC recruitment of a subcortical antinociceptive network. Pain 2006;120:8-15.

- 32 Zubieta JK, Bueller JA, Jackson LR, Scott DJ, Xu Y, Koeppe RA, Nichols TE, Stohler CS: Placebo effects mediated by endogenous opioid activity on $\mu$-opioid receptors. J Neurosci 2005;25:7754-7762.

33 Levine JD, Gordon NC, Fields HL: The mechanism of placebo analgesia. Lancet 1978;2:654-657.

- 34 Krummenacher P, Candia V, Folkers G, Schedlowski M, Schonbachler G: Prefrontal cortex modulates placebo analgesia. Pain 2010;148:368-374.

- 35 Benedetti F, Arduino C, Costa S, Vighetti S, Tarenzi L, Rainero I, Asteggiano G: Loss of expectation-related mechanisms in Alzheimer's disease makes analgesic therapies less effective. Pain 2006;121:133-144.

- 36 Petrovic P, Kalso E, Petersson KM, Ingvar M: Placebo and opioid analgesia - imaging a shared neuronal network. Science 2002;295: 1737-1740.

- 37 Price DD, Craggs J, Verne GN, Perlstein WM, Robinson ME: Placebo analgesia is accompanied by large reductions in pain-related brain activity in irritable bowel syndrome patients. Pain 2007;127:63-72.

- 38 Eippert F, Finsterbusch J, Bingel U, Buchel C: Direct evidence for spinal cord involvement in placebo analgesia. Science 2009;326:404.

39 Fields H: State-dependent opioid control of pain. Nat Rev Neurosci 2004;5:565-575.
40 Petrovic P, Dietrich T, Fransson P, Andersson J, Carlsson K, Ingvar M: Placebo in emotional processing-induced expectations of anxiety relief activate a generalized modulatory network. Neuron 2005;46:957-969.

41 Mayberg HS, Silva JA, Brannan SK, Tekell JL, Mahurin RK, McGinnis S, Jerabek PA: The functional neuroanatomy of the placebo effect. Am J Psychiatry 2002;159:728-737.

42 Leuchter AF, Cook IA, Witte EA, Morgan M, Abrams M: Changes in brain function of depressed subjects during treatment with placebo. Am J Psychiatry 2002;159:122-129.

43 Furmark T, Appel L, Henningsson S, Ahs F, Faria V, Linnman C, Pissiota A, Frans O, Bani M, Bettica P, Pich EM, Jacobsson E, Wahlstedt K, Oreland L, Langstrom B, Eriksson E, Fredrikson M: A link between serotonin-related gene polymorphisms, amygdala activity, and placebo-induced relief from social anxiety. J Neurosci 2008; 28:13066-13074.

44 Leuchter AF, McCracken JT, Hunter AM, Cook IA, Alpert JE: Monoamine oxidase and catechol-O-methyltransferase functional polymorphisms and the placebo response in major depressive disorder. J Clin Psychopharmacol 2009;29:372-377.

45 de la Fuente-Fernández R, Ruth TJ, Sossi V, Schulzer M, Calne DB, Stoessl AJ: Expectation and dopamine release: mechanism of the placebo effect in Parkinson's disease. Science 2001;293:1164-1166.

46 Benedetti F, Colloca L, Torre E, Lanotte M, Melcarne A, Pesare M, Bergamasco B, Lopiano L: Placebo-responsive Parkinson patients show decreased activity in single neurons of subthalamic nucleus. Nat Neurosci 2004;7:587-588.

47 Benedetti F, Lanotte M, Colloca L, Ducati A, Zibetti M, Lopiano L: Electrophysiological properties of thalamic, subthalamic and nigral neurons during the anti-parkinsonian placebo response. J Physiol 2009;587:38693883 .

48 Schweinhardt P, Seminowicz DA, Jaeger E, Duncan GH, Bushnell MC: The anatomy of the mesolimbic reward system: a link between personality and the placebo analgesic response. J Neurosci 2009;29:4882-4887.

49 Levine JD, Gordon NC: Influence of the method of drug administration on analgesic response. Nature 1984;312:755-756.

50 Amanzio M, Pollo A, Maggi G, Benedetti F: Response variability to analgesics: a role for non-specific activation of endogenous opioids. Pain 2001;90:205-215.

-51 Colloca L, Lopiano L, Lanotte M, Benedetti F: Overt versus covert treatment for pain, anxiety, and Parkinson's disease. Lancet Neurol 2004;3:679-684.
52 Lyerly SB, Ross S, Krugman AD, Clyde DJ: Drugs and placebos: the effects of instructions upon performance and mood under amphetamine sulphate and chloral hydrate. J Abnorm Psychol 1964;68:321-327.

53 Ulrich RS: View through a window may influence recovery from surgery. Science 1984; 224:420-421.

54 Kaptchuk TJ, Kelley JM, Conboy LA, Davis RB, Kerr CE, Jacobson EE, Kirsch I, Schyner RN, Nam BH, Nguyen LT, Park M, Rivers AL, McManus C, Kokkotou E, Drossman DA, Goldman P, Lembo AJ: Components of placebo effect: randomised controlled trial in patients with irritable bowel syndrome. BMJ 2008;336:999-1003.

55 Barsky AJ, Saintfort R, Rogers MP, Borus JF: Nonspecific medication side effects and the nocebo phenomenon. JAMA 2002;287:622627.

56 Rief W, Nestoriuc Y, von Lilienfeld-Toal A, Dogan I, Schreiber F, Hofmann SG, Barsky AJ, Avorn J: Differences in adverse effect reporting in placebo groups in SSRI and tricyclic antidepressant trials: a systematic review and meta-analysis. Drug Saf 2009;32:10411056.

57 Amanzio M, Corazzini LL, Vase L, Benedetti F: A systematic review of adverse events in placebo groups of anti-migraine clinical trials. Pain 2009;146:261-269.

58 Bodenheimer $\mathrm{T}$ : The future of primary care: transforming practice. N Engl J Med 2008; 359:2086, 2089.

59 Conboy LA, Macklin E, Kelley J, Kokkotou E, Lembo A, Kaptchuk T: Which patients improve: characteristics increasing sensitivity to a supportive patient-practitioner relationship. Soc Sci Med 2010;70:479-484

60 Sneed JR, Rutherford BR, Rindskopf D, Lane DT, Sackeim HA, Roose SP: Design makes a difference: a meta-analysis of antidepressant response rates in placebo-controlled versus comparator trials in late-life depression. Am J Geriatr Psychiatry 2008; 16:65-73.

- 61 Edwards RR, Bingham CO 3rd, Bathon J, Haythornthwaite JA: Catastrophizing and pain in arthritis, fibromyalgia, and other rheumatic diseases. Arthritis Rheum 2006; 55:325-332.

62 Colloca L, Benedetti F, Porro CA: Experimental designs and brain mapping approaches for studying the placebo analgesic effect. Eur J Appl Physiol 2008;102:371-380. 